\title{
Questes
}

\section{Le rapport au désuet et à l'obsolète à la fin du Moyen Âge : l'apport des sources urbaines}

Anne Kucab

\section{(2) OpenEdition}

Édition électronique

URL : http://journals.openedition.org/questes/5312

DOI : 10.4000/questes.5312

ISSN : 2109-9472

Éditeur

Les Amis de Questes

\section{Édition imprimée}

Date de publication : 28 février 2019

Pagination : $37-54$

ISSN : 2102-7188

\section{Référence électronique}

Anne Kucab, «Le rapport au désuet et à l'obsolète à la fin du Moyen Âge : l'apport des sources

urbaines », Questes [En ligne], 40 | 2019, mis en ligne le 19 avril 2019, consulté le 22 avril 2019. URL :

http://journals.openedition.org/questes/5312; DOI : 10.4000/questes.5312

Ce document a été généré automatiquement le 22 avril 2019.

(c) Association des amis de «Questes » 


\title{
Le rapport au désuet et à l'obsolète à la fin du Moyen Âge : l'apport des sources urbaines
}

\author{
Anne Kucab
}

1 Percevoir, au travers des sources, l'idée de désuétude ou d'obsolescence est un défi d'autant plus grand pour l'historien que le Moyen Âge ne faisait pas usage de ce vocabulaire. Pourtant, les hommes et les femmes du Moyen Âge étaient sensibles au passage du temps. L'un des meilleurs moyens d'appréhender ce phénomène est de s'intéresser aux termes marquant la perception du vieillissement et de l'usure des objets, donc de leur obsolescence. Le regard porté sur l'objet usé permet également d'aborder la conception de la désuétude. Les travaux récents sur la consommation ont souligné la place importante du marché de l'occasion dans la vie quotidienne, notamment pour les groupes sociaux urbains. Ces études montrent qu'un grand nombre de personnes possédaient des objets et des vêtements marqués d'un certain degré d'obsolescence en raison de leur usure ou de leur rapiéçage. Mais de tels biens, parfois de seconde main, étaient également perçus comme désuets en raison de leur style connotant l'ancien. La fin du Moyen Âge est bien sujette aux effets de mode qui établissent des distinctions entre un vêtement à la pointe de la nouveauté et des vêtements plus communs marqués par un style évoquant davantage le passé que l'avenir. Il semble toutefois difficile d'affirmer qu'au bas Moyen Âge, les « classes populaires » seraient vêtues de manière désuète quand les riches porteraient élégamment la dernière mode ${ }^{1}$.

2 Pour saisir le sentiment de l'obsolète et du désuet, nous nous concentrerons sur les sources urbaines de la fin du Moyen Âge, et notamment sur les sources de la pratique (comptabilités et délibérations municipales rouennaises, compte de Colin de Lormoye, tailleur parisien de la première moitié $d u \mathrm{xv}^{\mathrm{e}}$ siècle) que nous confronterons avec des testaments et des textes littéraires tels que ceux de Christine de Pizan. En raison de leur spécificité, les sources matérielles ne seront pas étudiées de manière détaillée. Soulignons toutefois que les archéologues mettent fréquemment au jour des objets dont la période d'usage est longue. Il faut donc garder en tête que la date de fabrication d'un objet 
n'augure en rien sa date d'utilisation: des monnaies ou des formes de céramiques peuvent être employées pendant plusieurs décennies ou plusieurs siècles. Les objets étaient ainsi réparés et utilisés autant que possible, avant d'être jetés comme en témoignent les dépotoirs médiévaux et modernes fouillés à Rouen² ou à Lyon ${ }^{3}$. À Rouen, ces heurts, pour reprendre leur désignation locale, ont été étudiés (en particulier ceux du $\mathrm{xVI}^{\mathrm{e}}$ siècle) par Bénédicte Guillot. Leur étude a mis en avant le fort recyclage et la longue utilisation du matériel avant que ce dernier ne soit jeté, car «la nature même d'un dépotoir implique que l'on n'y retrouve que des objets perdus, cassés ou devenus

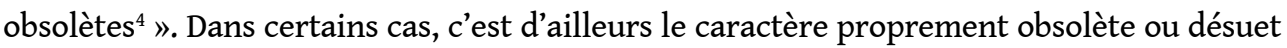
de ces objets qui conduit à leur thésaurisation.

3 Au cours des pages qui vont suivre, nous nous demanderons quels sont, dans les sources urbaines, les indices que l'historien peut interpréter comme des marques de désuétude et d'obsolescence des objets du quotidien. Dans un premier temps, nous nous intéresserons aux objets marqués par le temps, avant de montrer que cette usure suscite une volonté de les remettre au goût du jour. Enfin, nous nous interrogerons sur la perception du caractère ancien des objets dans les sources médiévales à travers deux exemples: les vêtements et les œuvres d'art d'une part (avec le phénomène de mode), la monnaie d'autre part.

\section{Des objets usés, obsolètes ou désuets}

\section{Des cas où l'objet ancien est perçu comme obsolète ou désuet ?}

Les objets tels que les vêtements subissent les outrages du temps et les différentes sources montrent que les médiévaux y sont sensibles. Revendre ou donner des vêtements devenus trop vieux ou usés étant au Moyen Âge une habitude bien ancrée, le marché de l'occasion est florissant et c'est même pour certains, comme les fripiers, un métier. De fait, en 14561457 , la ville de Rouen dispose d'une halle « aux chinchiers et aux pelletiers de vieil », où les vêtements et les fourrures d'occasion sont revendus, ainsi que des «lingeres de vieil $»^{5}$ . Les raisons qui poussent à mettre un objet ancien en vente ${ }^{6}$ sont multiples. Si la nécessité économique prévaut le plus souvent - il s'agit de faire rentrer de l'argent dans la bourse familiale -, nous devons aussi envisager une volonté de se débarrasser ou de recycler des objets jugés désuets ou démodés. Enfin, la circulation des objets usagés est aussi alimentée par les legs testamentaires, qui en permettent parfois la transmission sur plusieurs générations.

5 Les deux facteurs se cumulent parfois, comme lorsqu'il s'agit de vendre aux enchères les objets et vêtements du défunt afin de payer ses obsèques et ses dettes. Juan Vicente Garcia Marsilla, qui a étudié le phénomène à Valence en Espagne, montre bien le rôle important des pellers, revendeurs qu'il qualifie de "professionnels du rafistolage » ou " professionnels de l'usagé »". En raison de la nature de leur activité, ces individus sont particulièrement sensibles à l'usure du vêtement qu'ils doivent estimer et qu'ils décrivent parfois de manière très précise ${ }^{8}$. Les sources soulignent cette différenciation entre des degrés d'usure. Ainsi, les testaments rouennais conservés établissent des distinctions entre les vêtements en bon état, comme ceux que lègue Jeanne Boissel à ses descendants. Elle laisse en effet à sa fille " son bon chaperon ", à sa petite-fille " son meilleur chaperon après celui de sa mère ", et à d'autres bénéficiaires des atours usés comme un "viel queuvrechief $»^{9}$. De même, l'inventaire après décès de Denise de Foville dressé en 1465 
établit une différence entre "ung petit paire de draps à lits" pour la somme 10 sous, 2 deniers, " ung autre paire vieux » coûtant 10 sous, et "ung aultre paire plus vieulx » estimé à 6 sous 6 deniers ${ }^{10}$. D'autres pièces de l'inventaire sont également notées avec leur degré d'obsolescence, comme ce canevas (pièce de grosse toile) qualifié de « fort usé », ou cette " petite serviette usée », ou encore une vieille robe noire ${ }^{11}$. De manière générale, les vêtements les moins usés et les moins désuets sont légués aux proches quand les autres reviennent aux domestiques. Est-ce à dire que les moins fortunés étaient habillés de manière obsolète? Difficile de l'affirmer comme une vérité générale, mais Juan Vicente Garcia Marsilla en fait le constat pour Valence :

Comment distinguer à cette époque la garde-robe d'un potentat par rapport à celle des classes plus modestes? Les différences sont dans l'aspect visuel direct, par l'apparence d'un habit que l'on porte à un moment donné ou d'une manière beaucoup plus subtile sur la longue durée, en observant les vêtements portés à une certaine époque. Il reste donc à évaluer ces aspects, comme le nombre d'habits qui constituaient la garde-robe de chacun, la capacité à la renouveler et, par conséquent, la possibilité de suivre les changements de la mode ${ }^{12}$.

6 La perception de l'usure témoignant de la prise de conscience de l'obsolescence, c'est-àdire la perte des qualités premières d'un objet ${ }^{13}$, ou de la désuétude, entendue comme la non-concordance de son style ou aspect avec les attentes esthétiques et sociales, variait selon les milieux. Les catégories sociales les plus aisées cherchaient généralement à posséder des nouveautés ou tout du moins des objets neufs, qui reflétaient leur situation économique et leur statut social. On peut évoquer la mode du vêtement court pour les hommes qui apparaît au XIV ${ }^{e}$ siècle au détriment de la robe et qui, dans un premier temps, concerne essentiellement les plus fortunés ${ }^{14}$.

7 L'obsolescence et la désuétude ne concernent pas uniquement le vestiaire: le même phénomène s'observe à travers la revente de vieux objets, par exemple les œuvres d'art. En 1406-1407, la fabrique de la cathédrale de Rouen gratifie de 2 sous 6 deniers un peintre qui avait vendu une vieille image ${ }^{15}$. En 1444-1445, le trésorier de la paroisse Saint-Nicolas note un gain de 3 sous à la suite de la vente « d'un vieil ymage à gens de dehors la ville ${ }^{16}$ ". Le chapitre cathédral revend en mars 1459 plusieurs livres décrits comme anciens et inutiles ${ }^{17}$. Les sources laissent apparaitre la perception du vieillissement et de l'usure des objets qui amènent leurs propriétaires à les revendre, souvent pour pouvoir les remplacer par des objets plus neufs, comme c'est le cas pour les œuvres d'art. Faut-il y lire en filigrane que les contemporains jugeaient ces vieux objets désuets ou obsolètes?

\section{Vicissitudes politiques et obsolescence}

8 Si certains objets deviennent désuets à moyen terme en quelques décennies, d'autres sont frappés d'obsolescence du jour au lendemain. C'est le cas des éléments liés à l'appartenance politique qui deviennent obsolètes au premier changement de conjoncture. Nous avons conservé quelques traces de ces retournements de situation qui rendent caduc voire dangereux l'affichage de son opinion politique. Ainsi à Rouen, dans les dernières années de la guerre de Cent Ans, les comptes de l'archevêque pour l'année 1449-1450 montrent la déposition des verrières aux armes du roi d'Angleterre, devenues obsolètes suite au recouvrement de la ville par le roi de France Charles VII à l'automne 1449 : « [Recette] de la vendicion des verrières aux armes d'Engleterre qui ont eté ostées de la second salle de l'ostel, [e]t au lieu on a mis du verre blanc à la venue du roi ${ }^{18} »$. Elles 
sont remplacées par des verrières aux armes de l'archevêque au cours de l'année 1454$1455^{19}$.

Lors de la guerre civile entre Armagnacs et Bourguignons, les partisans des deux factions affichent leurs convictions au moyen d'enseignes : dauphin ou écu du Dauphin pour les Armagnacs, et rabot ou croix de Saint-André pour les Bourguignons ${ }^{20}$. Ces enseignes permettent de manifester son opinion tout en constituant également un signe de reconnaissance, voire une protection. Pourtant, lorsque la conjoncture évolue brutalement, leurs possesseurs s'empressent de faire définitivement disparaitre ces signes d'appartenance politique :

Le port de l'enseigne qui révélait une opposition au pouvoir en place était très dangereux, si bien qu'il fallait s'en débarrasser au plus vite. C'est peut-être pour cette raison que de nombreuses enseignes des Bourguignons et des Armagnacs ont été recueillies à Paris dans les alluvions de la Seine ${ }^{21}$.

En effet, les différents dragages de la Seine ont à ce jour permis de retrouver six enseignes du parti Armagnac et cinq du parti Bourguignon, ainsi que quatre enseignes aux armes de France $^{22}$. Elles témoignent des vicissitudes de la vie politique à la fin du Moyen Âge et du danger inhérent à l'obsolescence des signes d'appartenance politique ${ }^{23}$. Si les hommes et femmes du Moyen Âge avaient conscience de l'obsolescence, parfois brutale, des signes liés au pouvoir, ils souhaitaient aussi, dans certains cas, prolonger leur durée de vie autant que possible.

\section{Faire durer}

11 Les sources soulignent une volonté de faire perdurer l'usage de certains objets ou vêtements. D'abord d'ordre économique et fonctionnel - il s'agit de réparer, de rendre à nouveau utilisable, d'embellir l'objet -, ces pratiques sont aussi la marque d'un refus du désuet. En rénovant, réparant, réintégrant, les hommes du Moyen Âge luttent contre la désuétude et l'obsolescence.

\section{Nettoyer, réparer, retailler, enjoliver les vêtements}

Une source permet de bien saisir cette lutte contre la désuétude des objets: il s'agit d'extraits du livre de comptes d'un tailleur parisien, actif dans la première moitié du $\mathrm{Xv}^{\mathrm{e}}$ siècle, Colin de Lormoye ${ }^{24}$. Ce tailleur joue parfois les intermédiaires pour l'entretien des vêtements des plus riches et reçoit ainsi des vêtements à faire laver : «Item ay fait laver et nestier dux houpelendes noires pour luy, pour ce ii s. viij d. p..$^{25}$ », ou « Item pour laver la chapper et pour la defroucer par le frepier, ij s. p. ${ }^{26}$ ». Certains vêtements, probablement en drap de laine, lui sont également donnés pour être refoulés ${ }^{27}$ («Item pour maistre Simon ay fait reffouler une houpelande, viij s..$^{28} »$ ) ou reteints (« Item, [...] une robe retainte, pour la fason vi s. p..$^{29} »$ ). Ainsi, de nouveaux biens sont rendus disponibles à l'usage par le recyclage d'objets obsolètes.

Cependant, Colin de Lormoye ne taillait pas uniquement des habits neufs. Il intervenait également sur des habits anciens pour les réparer ou leur donner une seconde jeunesse. Cette part de travail n'est pas négligeable, puisqu'elle concerne presque $13 \%$ des 869 vêtements mentionnés dans le compte. L'essentiel des reprises concerne des robes ( $33 \%$ des reprises), des houppelandes ( $22,5 \%$ ) ou des pourpoints $(20,5 \%)$, habits coûteux dont la réparation est moins onéreuse que l'achat d'un habit neuf. Enfin, les chaperons 
(17 \%) sont bien représentés car il s'agissait de vêtements fréquemment portés et dont le style évoluait plus rapidement que la plupart des autres vêtements, ce qui contraignait leurs propriétaires à de nombreuses modifications ou accommodements ${ }^{30}$. De fait, la majorité des réparations a un coût inférieur ou égal à 4 sous, ce qui est moins cher qu'un vêtement neuf ${ }^{31}$.

Les vêtements étaient donnés à Lormoye pour être remis en état. Les variantes des syntagmes mettre à point ou remis à point, qui signifient que les vêtements sont " rafraichis ", sont très présentes dans les comptes. Il peut s'agir de refaire, de réparer ou de reparaisons, d'arranger, de rappareiller, de rappointer (c'est-à-dire « raccommoder »), de rabiliet (ou rhabiller, soit "réparer, remettre en état» en moyen français ${ }^{32}$ ). Toutes ces expressions plus ou moins synonymes de réparation, de raccommodage ou de remise à neuf, qui apparaissent sous de nombreuses variantes graphiques, montrent l'importance de l'activité de reprise des vêtements. Elles soulignent également une sensibilité à leur usure, donc à leur obsolescence, et une volonté de les remettre au goût du jour, ce qui dénote une perception de leur style désuet. Les rares indications contenues dans le compte nous permettent d'avoir une idée un peu plus précise des réparations effectuées par Colin de Lormoye.

ernier pouvait également « recycler » des vêtements, c'est-à-dire faire de nouveaux vêtements à partir d'anciens vêtements : faire une houppelande nouvelle à partir d'une ancienne, une robe d'un manteau éculé, un nouveau chaperon d'un ancien ${ }^{33}$, ou encore rallonger une manche ou une robe ${ }^{34}$. L'expression retourner un vêtement revient à de nombreuses reprises dans les comptes, et il semble qu'il s'agisse de découdre le vêtement pour mettre la face intérieure à l'extérieur. Cette pratique peut s'expliquer par la volonté de faire des économies : l'extérieur du vêtement étant trop usé, taché ou les couleurs ayant passé, il était plus simple d'inverser face extérieure et face intérieure pour avoir à nouveau un vêtement présentable tout en économisant le prix du tissu. Ce subterfuge semble avoir été très répandu, car il permettait de toujours "bien paraître » à moindre coût. Une telle pratique était peut-être anticipée dans la première confection de la pièce puisque certains vêtements (robes ou houppelandes) sont doublés d'un drap d'une couleur différente de celui se trouvant à l'extérieur ${ }^{35}$.

La réparation des vêtements constitue par conséquent une part non négligeable du travail de Colin de Lormoye. Quel que soit le rang social ${ }^{36}$, le vêtement, et surtout le drap, coûte cher; il faut donc en prendre soin et le rentabiliser au maximum tout en prêtant attention au paraître. Ces réhabilitations fréquentes soulignent aussi que les vêtements usés, élimés, voire désuets ne sont pas bien perçus et qu'il est nécessaire de les remettre au goût du jour pour éviter qu'ils ne deviennent obsolètes.

\section{Rénover les œuvres d'art}

Un phénomène similaire de réparation et de remise au goût du jour s'observe avec les œuvres d'art. Le 20 janvier 1455, le cardinal et archevêque de Rouen, Guillaume d'Estouteville, donne la permission de nettoyer et de redorer les images de Notre-Dame posées sur l'autel ${ }^{37}$; en 1462-1463, c'est l'image de Notre-Dame (en albâtre peint) figurant sur le tronc et l'image de la sépulture du roi qui sont nettoyées ${ }^{38}$. Cette politique d'entretien de la cathédrale se poursuit en 1463-1464 puisqu'on retire du portail aux libraires «des images» sur le point de tomber et qu'on nettoie «la passion entour le pipistre à l'entree du choeur ${ }^{39} »$. Certains tableaux nécessitent une véritable restauration 
effectuée par un spécialiste, comme en 1467-1468 où le dénommé Heuzé, peintre, reçoit 11 livres pour "peinture et réparation " d'une image de la chapelle Sainte-Anne de la cathédrale ${ }^{40}$. Un proche du trésorier, Jean Baron, se fait réprimander par le chapitre pour avoir placé les torches et les chandelles dans la chapelle de telle manière qu'elles abîmaient les peintures des murs et les images ${ }^{41}$. Nous retrouvons la même attention portée aux images dans les comptes du collège du Saint-Esprit en 1482, puisqu'un homme reçoit 15 deniers pour avoir «nestié les courtines et 2 images de la chapelle ${ }^{42}$ ». Le soin apporté à la conservation des images souligne là encore la conscience du temps qui passe et de ses méfaits: perte de couleur, suie. En effet, sans soin apporté à leur bonne conservation, il y a des risques que les objets deviennent obsolètes en ne pouvant plus remplir leurs fonctions votives. Cependant, la dégradation matérielle de l'objet n'est pas uniquement due au passage du temps car les effets de mode contribuent rapidement à donner un caractère désuet, et parfois même obsolète, aux objets.

\section{Entre obsolescence et réemploi}

\section{Mode et désuétude vestimentaires}

Les phénomènes de mode sont attestés tout au long du Moyen Âge et s'accélèrent de manière notable à partir $d u$ XIII $^{e}$ siècle ${ }^{43}$. Nous nous concentrerons donc sur la fin du Moyen Âge, période pour laquelle nous considérerons que la mode est l'émergence de préférences et de "normes" collectives qui sont en vigueur pour une durée plus ou moins longue. Si la fin du Moyen Âge est clairement marquée par des préférences collectives, notamment en termes de vêtements, peut-on pour autant parler de « mode " ? Le terme apparaît au cours du Moyen Âge et signifie dans un premier temps la «façon ». Puis, il prend le sens d'« innovation vestimentaire dans l'air du temps ${ }^{44}$ ». À la fin du Xve siècle, deux poèmes de Guillaume Coquillart font figurer le terme mode dans le sens d'« innovation vestimentaire qui rend désuètes les autres manières de s'habiller »: "Quelque une qui a fronc ridé /Porte devant une custode, /Et puis on dit qu'elle a cuidé / Trouver une nouvelle mode ${ }^{45} »$; « Tel fringue à la mode nouvelle ${ }^{46} »$. Le terme de mode est entré dans les esprits pour parler des changements vestimentaires.

Pour certains contemporains, la mode semble anachronique au sens où elle induit des normes nouvelles. Elle apparaît aussi comme une futilité ne s'inscrivant pas dans la durée et destinée à devenir rapidement désuète. Christine de Pizan a parfaitement décrit le phénomène grégaire qu'est la mode, preuve qu'en ville ce type de comportement existait dès le bas Moyen Âge :

pour ce yppert comment tout va: n'y a es abiz ne es attours regle tenue. Car qui plus en puet faire de quelque estat que ce soit, soient femmes ou hommes, leur semble qu'ilz besoingnent le mieulx, et tout ainsi que les berbis suivent l'une l'autre, s'il y a aucun homme ou femme qui voye faire a aultre quelque oultrage ou desordonnance en habit ou abillement, tantost les autres le suivent et dient il fault faire comme les aultres. Mais ilz dient voir : il fault que un oultrageux suive un aultre ${ }^{47}$.

Elle parachève sa critique sur le thème, depuis devenu célèbre, de l'éphémère de la mode :

Et cecy [les changements de vêtements] est quant aux dames de France, car es autres païs se tiennent plus longuement communement les coustumes qu'ont tant hommes comme femmes en leurs abillemens, non mie changent d'an en an, comme cil qui va tousjours en croiscent oultrages ${ }^{48}$. 
"Le processus de la mode" est précoce mais décrié, il ne s'agit pas de posséder la dernière nouveauté mais bien de l'éviter : «Si apertient doncques a toute femme qui veult garder bonne renommee que elle soit [...] $\mathrm{n}[\mathrm{on}]$ trop grant trouverresse de choses nouvelles, par especial cousteuses et non honnestes ${ }^{49} \%$. On observe une sorte de valorisation du style ancien face à un style nouveau : à l'inverse d'aujourd'hui, le désuet dans le vêtement est valorisé parce qu'incarnant la modestie, la conformité à son groupe social.

Les phénomènes de mode sont donc perçus comme incongrus par une partie des gens du Moyen Âge. L'habillement médiéval procède selon deux logiques : d'un côté, il est sans cesse réutilisé à travers le vêtement d'occasion et le raccommodage, il perdure donc sur un temps long; de l'autre, de nouvelles normes sociales le rendent rapidement périmé et désuet, il a alors une durée de vie beaucoup plus courte. Quoi qu'en dise Christine de Pizan, les travaux de Juan Vicente Garcia Marsilla confirment que ce phénomène n'est pas uniquement français et qu'il se retrouve aussi en Espagne :

Le marché de l'occasion se rapprocha peu à peu des nouveautés du costume pour les classes moyennes, et sûrement il créa de nouvelles perspectives de consommation pour celles-ci ainsi qu'un désir de suivre les modes lancées par les élites. [...] Le système devait donc fonctionner en suivant sa propre logique, c'est-à-dire en allongeant la vie des choses ${ }^{50}$.

\section{La monnaie, toujours réemployée?}

23 Le vêtement n'est pas le seul exemple d'objet du quotidien dont on peut étudier la perception du vieillissement et de l'usure: la monnaie constitue un autre exemple pertinent pour notre sujet. Celle-ci est en effet sujette à de nombreuses réglementations qui la dévaluent, la réévaluent et provoquent son obsolescence. Essayer de saisir le rapport des hommes et des femmes du Moyen Âge à la monnaie permet donc d'envisager leur rapport à l'obsolète et au désuet.

Les monnaies restent au Moyen Âge très longtemps en usage. Les numismates y sont particulièrement sensibles, au point que le degré d'usure est pour eux un objet d'étude : "[Une] méthode consiste à tenter de corréler le degré d'usure d'une monnaie avec sa durée de circulation. Par usure est entendue l'altération des reliefs de la monnaie du fait de sa manipulation ${ }^{51} »$. Nous savons que le cours des monnaies varie, certaines pouvant être interdites et mises hors d'usage. Les comptabilités rouennaises font écho à ce processus, comme en 1488-1489 où un paragraphe détaille l'ensemble de la dévaluation :

Diminucion de monnoie à cause des monnoies descriées par le Roi le lundi gras, $18^{\mathrm{e}}$ jour de février 1487 : 1'angle d'or receu pour 66 sous, baillé pour 56 ; un lion d'or receu pour 50 , baillé pour 45 ; monnoie de halbardes, sur 62 sous 3 deniers, perte de 13 sous 3 deniers ; 2 mailles de Saint-Andrieu receues pour 31 sous 3 deniers pièce, baillées pour 27 sous 6 deniers ; - 2 escus d'or receuz pour 36 sous 6 deniers pièce, baillées pour 35 sous; 1 escu au soleil receu pour 37 sous 6 deniers, baillé pour 36 sous 3 deniers; un ducat receu pour 42 sous 6 deniers baillé pour 37 sous 6 deniers ; - un quart de noble d'Edouard receu pour 22 sous 6 deniers, baillé pour 18 sous 9 deniers; une maille du Trect, receue pour 28 sous, baillée pour 25 sous ; un angle receu pour 66 sous 8 deniers, baillé pour 53 sous 4 deniers, un esgle pris pour 50 sous, baillé pour 35 sous ; -1 escu de Bretaigne baillé pour 34 sous, receu pour 36 sous 6 deniers; monnaie de lyars estranges à 3 deniers pièce, ne valent que 1 denier; onzains à 12 deniers pièce, pris pour 11 deniers; monnaie du pays de Picardie n'ayant plus cours ${ }^{52}$. 
De fait, les comptes des fabriques cathédrales et paroissiales font des listes des monnaies non courantes reçues en recette ou en dons (dans des troncs, lors de quêtes, etc.). En dehors de leur fonction comptable, ces listes de monnaies peuvent aussi indiquer que les Rouennais se débarrassaient des monnaies au faible cours ou obsolètes (et donc non acceptées dans les transactions marchandes) dans les troncs et bassines situées dans les églises pour les dons. Cela manifeste donc une conscience du cours des monnaies et de leur droit à être en circulation ou non.

Ces monnaies devenues obsolètes font l'objet d'un traitement particulier visant à les différencier et à montrer qu'elles n'avaient plus cours. Dans sa thèse consacrée aux usages des monnaies, Thibault Cardon a souligné les processus mis en œuvre pour identifier ces monnaies hors d'usage :

Cette pratique [le fait de cisailler, percer ou marteler une monnaie ou un jeton] n'a pas encore fait l'objet d'un examen minutieux, mais on y reconnaît communément des traces de "démonétisation». Une ordonnance monétaire de 1315 stipule par exemple que toutes les monnaies n'ayant pas cours doivent être percées [...]. Les monnaies fausses sont ainsi percées ou découpées, à la fois pour qu'elles ne puissent plus servir et pour que, étant exposées sur un comptoir, elles préviennent que le commerçant sait les reconnaître ${ }^{53}$.

Ainsi dans le cas de Rouen, les comptes de 1488-1489 font écho à l'ordonnance royale prise par Charles VIII en janvier et qui précise les monnaies autorisées et leur cours. Dans cet acte, il est spécifié la façon «de rendre obsolète » une monnaie : «Et, au regard de toutes autres monnoyes, tant d'or que d'argent, qui ne sont cy-dessus specifiées et declairées, nous en avons deffendu et deffendons le cours et mise, et voulons que dès-àprésent, elles soient cizaillées et mises au feu pour billon ${ }^{54}$ ». Il existe donc des processus officiels qui manifestent l'obsolescence des monnaies et condamnent ainsi leur usage.

Cela n'empêche pas pour autant la thésaurisation et la réutilisation de ces monnaies à d'autres fins. Juan Vicente Garcia Marsilla a ainsi trouvé, dans les sources valenciennes, la mention d'un collier fait de ducats vénitiens ${ }^{55}$. Les monnaies qui n'étaient plus en usage ont donc, dans certains cas, acquis une valeur esthétique, voire apotropaïque. Thibault Cardon a étudié deux monnaies anciennes réutilisées. Il s'agit d'un sesterce d'Antonin le Pieux, regravé au Moyen Âge en talisman ${ }^{56}$, et d'un florin d'Utrecht à l'effigie du Christ, transformé en médaille à l'époque moderne et retrouvé dans un couvent lillois ${ }^{57}$. Ces deux cas soulignent que le caractère désuet et obsolète d'un objet peut le transformer en objet de valeur ou d'attention.

Ainsi que nous l'avons souligné dans l'introduction, les termes de désuet et d'obsolète n'apparaissent jamais dans les sources médiévales. Pourtant, les hommes et les femmes du Moyen Âge avaient conscience de l'usure des objets et de leurs effets de mode. On observe ainsi des processus pour maintenir l'objet en circulation et en état le plus longtemps possible. À l'inverse, les caprices de la politique et de la mode rendent rapidement obsolètes et désuets certains objets. Comme le montrent les exemples cités, l'étude de l'obsolescence et de la désuétude gagne à être protéiforme, si ce n'est pluridisciplinaire. Aux protestations littéraires de Christine de Pizan répond l'archéologie ; en regard des comptabilités condamnant l'usage de monnaies interdites, la numismatique nous propose des usages sur le temps long. Une lecture minutieuse des sources nous offre un aperçu de cette lutte contre le temps qui passe, et qui fait qu'au Moyen Âge comme aujourd'hui, les «vieux » objets sont revendus, repeints et rapiécés pour substituer la mode à l'obsolescence. 


\section{NOTES}

1. Françoise Piponnier, Perrine Mane, Le Vêtement au Moyen Âge, Paris, Adam Biro, 1995 ; Pourpoint, mantel et chaperon. Se vêtir à la cour de Savoie (1300-1450), dir. Nadège Gauffre-Fayolle, Milano, Silvana editoriale, 2015.

2. Bénédicte Guillot, Le Heurt de Bouvereuil. Étude d'un dépotoir public du XVI ${ }^{\mathrm{e}}$ siècle à Rouen, Rapport de fouille archéologique, Cesson-Sévigné, INRAP Grand Ouest, 2015 ; Bénédicte Guillot, Les Abords du heurt du donjon de Rouen. Fossé, butte et dépotoir public, Rapport de fouille archéologique, Cesson-Sévigné, INRAP Grand Ouest, à paraître. Nous remercions chaleureusement Bénédicte Guillot pour nous avoir communiqué ces informations.

3. Alban Horry, «Lyon : un dépotoir du bas Moyen Âge sur la Presqu'île à Lyon ", Archéologie du Midi médiéval, t. 20, 2002, p. 164-168.

4. Bénédicte Guillot, "La gestion des déchets à Rouen $a u x v I^{\mathrm{e}}$ siècle, premiers résultats de la fouille préventive de deux "heurts" ou décharges publiques ", Les Nouvelles de l'archéologie, n 151, 2018, p. 16-21, cit. p. 18.

5. Archives départementales de Seine-Maritime (désormais abrégées en ADSM), 3E1/ANC/XX2, comptes de l'année 1456-1457, non folioté.

6. Les mécanismes de mise en gage sont eux mieux connus, comme le montrent les travaux de Julie Claustre (notamment « Objets gages, objets saisis, objets vendus par la justice à Paris [XIv $\mathrm{e}_{-}$ $\mathrm{XV}^{\mathrm{e}}$ siècle] », dans Objets sous contraintes. Circulation des richesses et valeur des choses, dir. Laurent Feller et Ana Rodriguez, Paris, Publications de la Sorbonne, 2013, p. 385-402 ; Dans les geôles du roi. L'emprisonnement pour dette à Paris à la fin du Moyen Âge, Paris, Publications de la Sorbonne, 2007) ou ceux de Daniel Smail (Legal Plunder: Households and Debt Collection in Late Medieval Europe, Cambridge, Massachussetts, 2016).

7. Juan Vicente Garcia Marsilla, «Avec les vêtements des autres, le marché du textile d'occasion dans la Valence médiévale ", dans Objets sous contrainte, circulation des richesses et valeur des choses au Moyen Âge, op. cit., p. 123-143, cit. p. 139 et 140.

8. «Une bonne partie de ces pièces, entre 70 et $80 \%$, est décrite grâce à l'utilisation d'un adjectif péjoratif, comme sotil (de peu de valeur), oldà (en mauvais état), squinçat (effiloché) ou simplement vell (vieux)» (ibid., p. 125).

9. ADSM, G. 298. En 1476, Laurent Surreau lègue à son neveu « deux de [ses] meilleures robes à son choix » (Inventaire de Pierre Surreau, receveur général de Normandie [fait par Guillaume de La Fontaine] ; suivi du Testament de Laurens Surreau [14 août 1476] ; et de l'Inventaire de Denise de Foville [prieure de Saint-Paul-lès-Rouen], éd. J. Félix, Rouen, Lestringant, 1892, p. 91).

10. Ibid., p. 227.

11. Ibid., p. 227-230. Pour plus de précisions, se reporter à Anne Kucab, «Les circulations du vêtement en ville à la fin du Moyen Âge ", communication prononcée lors du colloque «Le vêtement au Moyen Âge : de l'atelier à la garde-robe ", Paris, 27 et 28 septembre 2016, organisé par le GAM-EHESS, L'Atelier du Centre de recherches historiques, à paraître.

12. Juan Vicente Garcia Marsilla, « Avec les vêtements des autres... », art. cit., p. 132.

13. Voir l'introduction de la présente revue. Comme les coordinateurs du numéro, nous avons considéré comme obsolète un objet qui tend à perdre sa qualité première parce qu'usé ou abîmé. Un vêtement usé sera celui qui ne protège plus du froid, tandis que dans le cas d'un vêtement désuet, c'est la perception qu'on en a qui compte. Son style, apparaissant par exemple comme « vieillot », pourra également être perçu comme un marqueur social. 
14. François Boucher, «Les conditions de l'apparition du costume court en France vers le milieu du XIV ${ }^{\mathrm{e}}$ siècle ", dans Recueil de travaux offerts à Clovis Brunel, Paris, Société de l'École des chartes, 1955, p. 183-192.

15. ADSM, G. 2481.

16. ADSM, G. 7323. Il s'agit de la paroisse Saint-Nicolas de Rouen.

17. ADSM, G. 2135.

18. ADSM, G. 48, compte de l'année 1449-1450.

19. ADSM, G. 52, compte de l'année 1454-1455.

20. On trouve également, au cours de la guerre de Cent Ans, l'utilisation du léopard ou de la fleur de lys sur des enseignes pour manifester sa sympathie au roi d'Angleterre ou de France.

21. Denis Bruna, Saints et diables au chapeau. Bijoux oubliés du Moyen Âge, Paris, Éditions du Seuil, 2007, p. 72.

22. Décompte établi à partir de l'inventaire fait par Denis Bruna, dans Enseignes de pèlerinages et enseignes profanes, Paris, Réunion des musées nationaux, 1996, p. 278-286.

23. Précisons que ce n'est pas l'unique explication: «Les raisons de la présence des enseignes françaises dans la Seine sont sans doute diverses. Ces objets ont pu être jetés parce que devenus inutiles, à moins que leurs propriétaires les aient délibérément lancés dans le fleuve pour espérer l'accomplissement d'un vœu: "le sacrifice" de petits effets personnels matérialisait souvent un geste votif. Cependant, dans le contexte de la guerre civile, les possesseurs d'enseignes politiques ont pu aussi s'en débarrasser rapidement au moment d'un changement de gouvernement car il était dangereux pour leur vie d'afficher ouvertement une opinion " (Denis Bruna, Enseignes de plomb et autres menues chosettes du Moyen Âge, Paris, Éditions du Léopard d'Or, 2006, p. 123).

24. Ce compte retrouvé dans une reliure a été publié par Camille Couderc au début du $x x^{\mathrm{e}}$ siècle : "Les comptes d'un grand couturier parisien $\mathrm{du} \mathrm{xv}^{\mathrm{e}}$ siècle ", Bulletin de la société de l'Histoire de Paris et de l'île-de-France, t. 38, 1911. Julie Claustre a intégralement repris ce dossier en rééditant le texte (notamment dans une version électronique) et en l'étudiant dans le cadre de son HDR : Julie Claustre, Transactions et institutions. Une histoire sociale de l'économie médiévale. Mémoire inédit: mémoires d'un artisan parisien $d u X^{e}$ siècle, Université Paris 1 Panthéon-Sorbonne, printemps 2018, garant Laurent Feller, soutenu le 3 juin 2018. Voir notamment le chap. IV, « Colin de Lormoye en ses "besognes": un professionnel du vêtement ", p. 290-365. Cette communication s'appuie en partie sur nos travaux de Master 2 de recherche: Se vêtir en ville à la fin du Moyen Âge. Entre production, consommation et exhibition, sous la direction de Mme Elisabeth Crouzet-Pavan, Université Paris IV-Sorbonne, 2012. Par souci de cohérence avec notre travail de Master et de commodité, nous avons choisi de conserver pour cet article les références à l'édition établie par Camille Couderc dans son article, en citant les numéros de compte établis par ce dernier, et non les numéros de pages. Nous ne pouvons toutefois qu'inciter à l'utilisation de la nouvelle édition établie par Julie Claustre, plus complète et moins fautive. De même, nous conservons l'appellation de compte mais souscrivons entièrement à l'usage du terme de papier. Tous nos remerciements vont à Julie Claustre pour nous avoir communiqué ces éléments.

25. Camille Couderc, «Les comptes d'un grand couturier parisien... », art. cit., compte $n^{\circ} 128$ de Mons. de Saint-Simon. Voir aussi les comptes n 84, 164 et 175.

26. Ibid., compte $\mathrm{n}^{\circ} 94$ : compte de Jehan Boucart.

27. Le foulage est une opération de draperie qui consiste à battre, presser le drap pour resserrer les fibres de la laine et donner plus de corps au tissu.

28. Camille Couderc, « Les comptes d'un grand couturier parisien... », art. cit., compte $n^{\circ} 102$.

29. Ibid., compte $\mathrm{n}^{\circ} 174$.

30. Pourpoint, mantel et chaperon. Se vêtir à la cour de Savoie (1300-1450), op. cit. Voir notamment p. 47-51. 
31. À titre indicatif, une houppelande coûte majoritairement 6 sous dans les comptes de Colin de Lormoye.

32. Nous avons fondé notre distinction orthographique et nos définitions sur le dictionnaire du moyen français mis en ligne par le département d'analyse et de traitement informatique de la langue française (ATILF) : http://www.atilf.fr/dmf/.

33. Camille Couderc, "Les comptes d'un grand couturier parisien...», art. cit., par exemple compte $n^{\circ} 25$ : «Item, une houpelande noyre, faite d'une hopelande d'écolier », compte $n^{\circ} 48$ : «Item, pour le dit enfant, ung chaperon découpé et decopé en un autre chaperon... », ou encore compte $n^{\circ} 163$ : «Item, pour ung aultre jeune Allement ay fait une houpelene noire d'un mentheau, pour la façon vj s. p.».

34. Ibid., compte $\mathrm{n}^{\circ} 179$ : « ralongiet les manches ».

35. Une autre mention précise : «item, luy ay retrouné une robe le devant darié é prins unes manches sur le cors, pour ce, v[j] s. p.» (ibid., compte $n^{\circ} 174$ ).

36. Colin de Lormoye habitait dans le Quartier latin, sa clientèle était donc composée de membres du clergé et de l'université pour plus d'un quart des professions mentionnées. Certains clients sont également identifiés comme noble, bourgeois, artisan ou commerçant, voire membre $\mathrm{du}$ parlement. Pour une analyse plus complète, voir Julie Claustre, Transactions et institutions, op. cit., « Une économie segmentée en plusieurs groupes d'acteurs », p. 321-332.

37. ADSM, G. 2135, 1454-1460.

38. ADSM, G. 2497, 1462-1463.

39. ADSM, G. 2499, 1463-1464.

40. ADSM, G. 2926, 1467-1468. L'année précédente, Roger le Maître avait reçu 3 sous pour la réparation d'un tableau placé dans le chapitre (ADSM, G. 2925, 1466-1467). Et en 1439-1440, Jehan Coquet avait également reçu 3 sous pour la réparation de tableaux placés dans le chapitre (ADSM, G. 2917, 1439-1447).

41. ADSM, G. 2137, 1465-1467.

42. ADSM, G. 4856, 1479-1482. Une opération semblable avait déjà été faite en 1467, puisque les images de la chapelle du Saint-Esprit avaient été nettoyées pour 15 deniers la veille de la Pentecôte, et en 1470 puisque les images de la chapelle sont à nouveau nettoyées et que « le tablel où est la représentation du crucifix » est « mis appoint » (ADSM, G. 4859, 1467-1470).

43. Michel Pastoureau et Odile Blanc, entre autres, datent l'apparition de la mode au XIII ${ }^{\mathrm{e}}$ siècle. Odile Blanc, "Le luxe, le vêtement et la mode à la fin du Moyen Âge », Bulletin du centre d'histoire économique et sociale de la région lyonnaise, 4, 1983, p. 23-44, et Parades et Parures. L'invention du corps de mode à la fin du Moyen Âge, Paris, Gallimard, 1997; Michel Pastoureau, "Le temps mis en couleurs : des couleurs liturgiques aux modes vestimentaires ( $\mathrm{XII}^{\mathrm{e}}-\mathrm{XIII}{ }^{\mathrm{e}}$ siècle) ", Bibliothèque de l'École des chartes, vol. 157, nº 1, 1999, p. 111-135.

44. Voir l'entrée « mode » du DMF, 2015.

45. Poème "Les droits nouveaux ", dans Guillaume Coquillart, Euvres, éd. Michelle Freeman, Genève, Droz, 1975, v. 1767-1770, p. 219.

46. Poème "Le debat des dames et des armes ", dans ibid., v. 424, p. 267. Le passage complet est le suivant: "Aux huis infinez fringuereaulx, /chascun soustenant sa querelle /Telle ayme ung tel, tel une telle, /Telle a promis/telle se plainct/tel fringue a la mode nouvelle/tel est ruzé, telle se faint $»$.

47. Christine de Pizan, Le Livre des trois vertus, éd. Éric Hicks et Charity Cannon Willard, Paris, Champion, 1989, Partie II, chap. 11, p. 157-158 (Christine de Pizan, Le Livre des trois Vertus, dans Voix de femmes au Moyen Âge. Savoir, mystique, poésie, amour, sorcellerie [XII ${ }^{e}-X V^{e}$ siècle], éd. Danielle $\mathrm{R}$ égnier-Bohler, Paris, Robert-Laffont, coll. «Bouquins», 2006, p. 655 : «Aussi voit-on comment tout va : en matière d'habits et de parure, on ne respecte aucune règle. Car ceux qui sur ce point peuvent surenchérir, quelle que soit leur condition et qu'ils soient hommes ou femmes, s'imaginent bien faire. À la façon des brebis qui emboîtent le pas à celles qui précèdent, il n'est 
pas d'homme ou de femme qui ne voie quelqu'un se livrer à quelque extravagance ou dérèglement dans sa mise ou sa façon de s'habiller sans le suivre aussitôt en déclarant qu'il faut faire comme les autres. Ils ont raison : il faut qu'un extravagant en imite un autre »).

48. Christine de Pizan, Le Livre des trois vertus, éd. cit., p. 159 (Voix de femmes au Moyen Âge, op. cit., p. 656 : «Tout ceci vaut pour les dames de France, car dans les autres pays les façons qu'ont les hommes et les femmes de s'habiller sont conservées généralement plus longtemps et ne changent pas chaque année pour aller toujours vers plus d'extravagance »).

49. Christine de Pizan, Le Livre des trois vertus, éd. cit., Partie II, chap. 2, p. 178 (Voix de femmes au Moyen Âge, op.cit., p. 668: «Toute femme qui veut conserver une bonne renommée [...] ne cherchera pas à inventer des modes nouvelles, surtout si elles sont coûteuses et peu décentes »). Voir dans le même ouvrage, Partie II, chap. 1, p. 176 : «Si fera tant que elle ait de tres beau linge delié [...] ; si le tendra blanc et souef flairant, bien ployé en coffres, et de ce sera tres soingneuse. [...] Ceste sage femme se prendra bien garde que riens ne pourrisse avaul son hostel ne voise a gast, de quoy povres creatures se puissent aucunement aidier ; [...] ne robes n'y soient mangies de vers : si les fera donner aux povres ».

50. Juan Vicente Garcia Marsilla, « Avec les vêtements des autres... », art. cit., p. 143.

51. Thibault Cardon, Les Usages des monnaies (mi-XII ${ }^{e}$-début $\mathrm{XVI}^{\mathrm{e}}$ siècle). Pour une approche archéologique, anthropologique et historique des monnaies médiévales, Thèse de doctorat sous la direction de Mathieu Arnoux et Marc Bompaire, École des hautes études en sciences sociales, Paris, soutenue le 4 mars 2016, p. 50.

52. Nous soulignons. ADSM, G. 2513 (1488-1489). En 1486-1487, le trésorier de la paroisse de Saint-Martin-du-Pont notait déjà les fluctuations de monnaie : «Pour ce que les grans blancs de Bourbon qui avoient cours eu président du jour d'uy pour 11 deniers et les ducas qui avoient cours pour 42 sous 6 deniers, lesquels grans blans furent diminuez à 10 deniers et les dits ducas à 42 sous 6 deniers, perte de 10 sous 7 deniers. ", ADSM, G. 3033 (1486-1487).

53. Thibault Cardon, Les Usages des monnaies, op. cit., p. 161.

54. Nous soulignons. Cette ordonnance défend également aux habitants du royaume l'usage des monnaies interdites sous peine de sanction. Ordonnances des rois de France de la troisième race, recueillies par ordre chronologique. Vingtième volume contenant les ordonnances rendues depuis le mois d'avril 1486, jusqu'au mois de décembre 1497, éd. Emmanuel Pastoret et Claude Arthus-Bertrand, Paris, Imprimerie Royale, 1840, « Ordonnance sur la monnaie », p. 56-58, cit. p. 57.

55. Juan Vicente Garcia Marsilla, « Avec les vêtements des autres... », art. cit., p. 132.

56. Thibault Cardon, "Un sesterce d'Antonin le Pieux regravé en talisman (XII $-\mathrm{XIV}^{\mathrm{e}}$ siècle) », Bulletin du cercle d'études numismatiques, vol. 54, n 2, mai-août 2017, p. 30-31.

57. Thibault Cardon et Christine Cercy, «Een Utrechtse gulden van bisschop Frederik van Baden tot penning gemaakt, Gevonden in Lille (Frankrijk) », De Beeldenaar, mars/avril 2018, p. 102-104.

\section{INDEX}

Keywords : Appearances, Coat of arms, Clothes, Custom, Clothing, Creation, Destruction 
AUTEUR

ANNE KUCAB

Sorbonne Université - Faculté des Lettres 